\title{
Temperature-independent slow carrier emission from deep-level defects in p-type germanium
}

\author{
S H Segers ${ }^{1}$, J Lauwaert ${ }^{1}$, P Clauws ${ }^{1}$, E Simoen ${ }^{2}$, J \\ Vanhellemont $^{1}$, F Callens ${ }^{1}$ and $\mathbf{H}$ Vrielinck $^{1}$ \\ ${ }^{1}$ Department of Solid State Sciences, Ghent University, Krijgslaan 281-S1, 9000 \\ Gent, Belgium \\ 2 IMEC, Kapeldreef 75, 3010 Leuven, Belgium \\ E-mail: henk.vrielinck@ugent.be
}

\begin{abstract}
In the deep-level transient spectroscopy (DLTS) spectra of the 3dtransition metals cobalt and chromium in p-type germanium, evidence is obtained that hole emission from defect levels can occur by two parallel paths. Besides classical thermal emission, we observed a second, slower and temperature-independent emission. We show that this extra emission component allows to determine unambiguously whether or not multiple DLTS peaks arise from the same defect. Despite similar characteristics, we demonstrate that the origin of the non-thermal emission is not tunneling but photoionization related to black body radiation from an insufficiently shielded part of the cryostat.
\end{abstract}

PACS numbers: 71.55.Cn, 72.20.Jv, 73.40.Gk

Submitted to: J. Phys. D: Appl. Phys. 


\section{INTRODUCTION}

Due to its high carrier drift mobility, Ge is used in advanced electronic devices. This has caused a revival of the interest in the electronic properties of impurities and intrinsic defects in Ge. A powerful technique to study these properties is Deep Level Transient Spectroscopy (DLTS). [1] Its high sensitivity and resolution have made it one of the most popular techniques for the characterisation of deep levels in semiconductors. In the last decades, DLTS has been used for a comprehensive study of metal impurities in Ge. The first succesful studies were accomplished on metal-diffused $\mathrm{Ge}(\mathrm{Cu}, \mathrm{Ag}$, $\mathrm{Au}$ and $\mathrm{Ni}$. 2 2 5ecause in in-diffused samples the contamination level of $\mathrm{Cu}$ and $\mathrm{Ni}$ is usually very high, the spectra of other transition metals ( $\mathrm{Ti}, \mathrm{Cr}, \mathrm{Fe}$ and $\mathrm{Co}$ ) were obtained on metal-implanted Ge. [6 9] All these impurities form multiple acceptors in agreement with occurence on substitutional lattice sites. For all acceptor levels observed in p-type material, field enhanced hole emission was observed except for $\mathrm{Co}^{0 /-}$ and $\mathrm{Fe}^{0 /-}$. Recently Gurimskaya et al. [10 claimed to have observed a Poole-Frenkel effect for hole emission via Minority Carrier Transient Spectroscopy on Fe in n-type Ge, which is not in agreement with the observation in p-type. [9] The shift observed by Gurimskaya et al. could, however, be influenced by a continuum of states that seems to be difficult to avoid in Schottky barriers. [10] The hole emission from the donor levels $\left(\mathrm{Co}^{0 /+}, \mathrm{Cr}^{0 /+}, \mathrm{Ag}^{0 /+}, \mathrm{Au}^{0 /+}\right)$ close to the valence band showed no observable field enhancement. [5, 8, 9] The agreement of the metal levels observed in DLTS with corresponding levels from Hall effect measurements [11] is excellent and the assignment to charge states seems rather well established. [5, 8, 99 The absence of field enhanced emission in the case of $\mathrm{Co}^{0 /-}$ and $\mathrm{Fe}^{0 /-}$ therefore indicates that it may not always be straightforward to conclude about the charge state of a level from the behaviour of carrier emission in presence of a strong electric field. In addition, although in the case of the metal centers the multiple acceptor nature was strongly supported by essentially equal concentrations of different levels, DLTS by itself is unable to decide in a straightforward way whether two levels are associated with the same defect. In all mentioned studies, interpretation of the observed capacitance transients relied on the assumption of thermally activated emission of carriers from deep levels. In this paper we report on a second, slow and temperature-independent emission component, observed in DLTS spectra of Co- and Cr-implanted Ge. Its spectral characteristics are similar to those of a parallel carrier emission path reported for quantum wells and dots in III-V semiconductors $[12-19]$ that was assigned to direct, through barrier tunnelling. The hole emission component reported here is, however, much slower and only becomes apparent in the spectrum when recording transients with large observer window time $\left(t_{W}\right)$.

The paper is further organized as follows. Section 2 describes the experimental details. In Section 3.1 the general characteristics of the additional emission component are illustrated for hole emission from the $\mathrm{Co}^{+/ 0}$ donor level. We show that this temperature-independent emission allows to study the hole capture characteristics in an extended temperature range. 
In section 3.2 the case of $\mathrm{Ge}$ : $\mathrm{Cr}$ is discussed, where similar temperature-independent emission is observed from two acceptor levels. The spectra allow to establish unambiguously that the two levels are related to the same defect. The electric field dependence and possible origin of the emission are discussed in Section 3.3. The conclusions are summarized in Section 4

\section{EXPERIMENTAL DETAILS}

The starting materials were p-type single crystal Ge wafers with a Ga shallow acceptor concentration of $1 \times 10^{14} \mathrm{~cm}^{-3}$, supplied by Electro-Optic Materials, Umicore. These wafers were implanted with $\mathrm{Co}$ or $\mathrm{Cr}$ at an energy of $90 \mathrm{keV}$ with a dose of $5 \times 10^{13}$ $\mathrm{cm}^{-2}$, as described elsewhere. [6, 8, 9] In order to remove the implantation damage and to diffuse the metal impurities deeper into the substrate, a 5 min post-implantation anneal at $500^{\circ} \mathrm{C}$ was applied in a $\mathrm{N}_{2}$ ambient. The samples were prepared for DLTS measurements by evaporating In to form a Schottky junction. This evaporation was preceded by a short etch in a $\mathrm{HNO}_{3}$ : $\mathrm{HF}$ (3:1) solution. Ohmic contacts were prepared using In-Ga eutectic and In foil. Our previous DLTS studies on these samples have shown that they exhibit no signals of residual implantation defects. The transition metal concentration in these samples near the junction is of the order $5 \times 10^{12} \mathrm{~cm}^{-3}$ and exhibits a slight in-diffusion profile. Capacitance DLTS measurements were performed with a Fourier transform instrument (Phystech FT1030) equipped with a Boonton 72B capacitance meter with an AC test signal of $1 \mathrm{MHz}$. The sample was placed in a Heraeus contact gas liquid He cryostat, shielded from the environmental black-body radiation by four heat shields. Extra shielding in the experiments described in Section 3.3 was provided by a $50 \mu \mathrm{m}$ aluminium foil covering the sample space.

In the DLTS measurements, capacitance transients were recorded at $V_{R}$ after a filling pulse $(200 \mathrm{~ms})$ to $\mathrm{V}_{P}\left(\mathrm{~V}_{R}<\mathrm{V}_{P}<0\right)$ and a waiting time $\mathrm{t}_{0}$, typically $\mathrm{t}_{W} / 512$ for temperature sweeps and $t_{W} / 4$ for isothermal measurements. In all DLTS spectra in this paper, the $\mathrm{b}_{1}$ Fourier component is shown:

$$
b_{1}=\frac{2 A}{t_{W}} \exp \left(-\frac{t_{0}}{\tau}\right)\left[1-\exp \left(-\frac{t_{W}}{\tau}\right)\right] \frac{\omega}{\frac{1}{\tau^{2}}+\omega^{2}},
$$

with $A$ the amplitude of the transient, $\tau$ the emission time constant and $\omega=\frac{2 \pi}{t_{W}}$.

Electric field values were calculated as described by Blood and Orton [20], assuming an abrupt depletion.

\section{EXPERIMENTAL RESULTS AND DISCUSSION}

\subsection{EXTRA EMISSION COMPONENT IN A SINGLE DLTS PEAK}

The DLTS spectrum of Ge:Co in the $30-180 \mathrm{~K}$ temperature range with $\mathrm{t}_{W}=5 \mathrm{~ms}$ is shown in figure 1(a). It features two peaks, corresponding to the $\mathrm{Co}^{+/ 0}$ level $\left(E_{A}=83\right.$ $\left.\mathrm{meV}, K_{T}=4.2 \times 10^{8} \mathrm{~s}^{-1} \mathrm{~K}^{-2}\right)$ and the $\mathrm{Co}^{0 /-}$ level $\left(E_{A}=254 \mathrm{meV}, K_{T}=1.7 \times 10^{7} \mathrm{~s}^{-1} \mathrm{~K}^{-2}\right)$, in agreement with Lauwaert et al. 9 

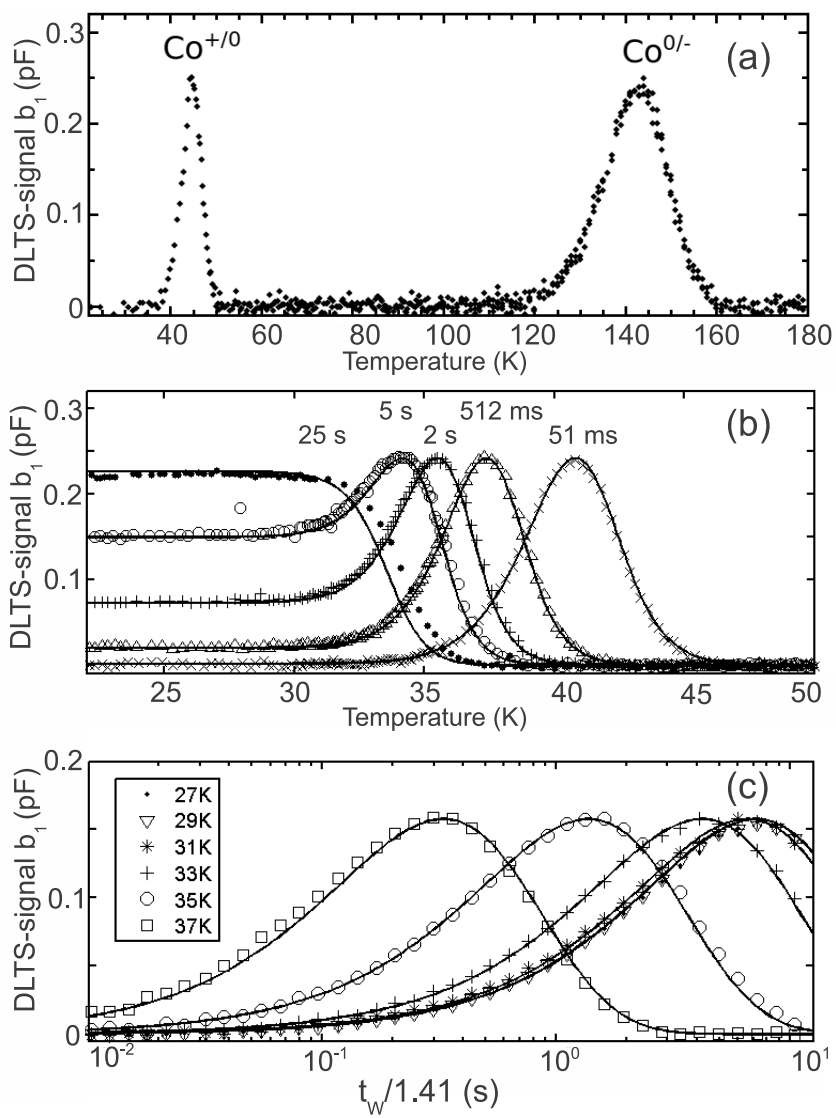

Figure 1. DLTS Spectra of Co in p-type Ge $\left(V_{R}=-2 \mathrm{~V}, V_{P}=-0.2 \mathrm{~V}\right)$ (a) Temperature scan at $t_{W}=5.12 \mathrm{~ms}$. (b) Influence of window time $t_{W}$ on the observation of the extra component of the $\mathrm{Co}^{+/ 0}$-peak in temperature sweep. (c) Isothermal DLTS measurements at several temperatures. The abscis represents $t_{W} / 1.41$ in order to allow for direct evaluation of the emission time constant $\tau_{\text {meas }}$ from the max of the spectra. Symbols represent experimental data points. The full lines are simulations based on equations 2 and 3 .

The $b_{1}$ Fourier DLTS spectrum of the $\mathrm{Co}^{+/ 0}$ peak in p-type Ge is shown in figure 1(b), recorded for a wide range of rate windows. At temperatures lower than the thermal emission temperature, an emission component with constant amplitude and emission time constant $\left(\tau_{c}\right)$ is observed for $t_{W}>51 \mathrm{~ms}$. This extra component is "shunted" by the thermal emission: after the thermal emission peak the $b_{1}$ amplitude falls to zero.

Figure 1 (c) displays isothermal DLTS spectra of this peak, recorded at different temperatures. These are all chosen such that thermal hole emission from $\mathrm{Co}^{0 /-}$ is too slow to appear in the experiment. At $\mathrm{T}>31 \mathrm{~K}$, the maximum shifts to lower values when $\mathrm{T}$ rises, as expected for a thermally activated process. Below $31 \mathrm{~K}$ there is no further shift of the maximum. However, the amplitude is temperature-independent in the whole temperature range. One can thus conclude that this type of emission affects all Co centers of this type (substitutional Co), not only those in a certain region or specific defect configuration. This yields two parallel emission paths for the holes: the 
"usual" thermal emission $\left(e_{t h}\right)$ and a second, temperature-independent emission $\left(e_{c}\right)$.

The total measured emission rate can be written as

$$
e_{\text {meas }}=e_{t h}+e_{c}
$$

with the thermal emission rate given by

$$
e_{t h}=K_{T} T^{2} \exp \left(\frac{-E_{a}}{k_{B} T}\right)
$$

with $k_{B}$ the Boltzmann constant. $K_{T}$, the pre-exponential factor and $E_{a}$, the apparent activation energy, constitute the signature of the defect level. The measured time constant $\tau_{\text {meas }}$ can be written as:

$$
\tau_{\text {meas }}=\frac{\tau_{c} \tau_{\text {th }}}{\tau_{c}+\tau_{\text {th }}}
$$

with $\tau_{c}=e_{c}^{-1}$, the temperature-independent time constant, and $\tau_{t h}=e_{t h}^{-1}$.

The full lines in figures 1(b) and (c) represent calculated fits to the experimental spectra, corresponding with $b_{1}$-Fourier transforms of single exponential capacitance transients with a time constant as in equation 4. The signature $\left(\mathrm{K}_{T}, \mathrm{E}_{A}\right)$ values were found from fitting and agree within experimental accuracy with those obtained from Arrhenius diagrams. 99 The excellent agreement with the experimental data justifies the analysis procedure and $\tau_{c}=7.3 \pm 0.7 \mathrm{~s}$ is found. This agreement also applies to other $\mathrm{a}_{n}$ and $\mathrm{b}_{n}$ Fourier components with the same value of $\tau_{c}$, demonstrating the closely exponential nature of the emission transient.

The extra emission component from a certain defect level can only be observed (at temperatures below the thermal peak of that level) when $t_{W}$ is sufficiently close to $\tau_{c}$ (up to about one order of magnitude different). For $t_{W}$ up to $25 \mathrm{~s}$, no extra emission component was observed for the $\mathrm{Co}^{0 /-}$ level. We estimate that if such emission would occur from this level, its time constant $\tau_{c}$ should exceed $300 \mathrm{~s}$.

Our previous DLTS study of Ge:Co has shown that capture kinetics of the $\mathrm{Co}^{+/ 0}$ level are sufficiently slow to allow for accurate determination of the capture cross section $\sigma$. The observation of an additional emission hence widens the temperature range in which $\sigma$ can be studied. Between 20-30 K, we found a fairly constant value of $(4.2 \pm 0.5) \times 10^{-14} \mathrm{~cm}^{2}$, in very good agreement with the values extracted from the thermal emission component. At these low temperatures, the capacitance transients need to be measured over long times $(\sim 1 \mathrm{~min})$, making these measurements more susceptible to noise and at the same time limiting the number of data points that can be taken for determining $\sigma$. As a result, the error on $\sigma$ is considerably larger than when measured via the thermal emission component. 21]

\subsection{EMISSION FROM TWO LEVELS OF THE SAME DEFECT}

In the previous section the additional temperature-independent emission from one defect level was observed $\left(\mathrm{Co}^{+/ 0}\right)$. In this section we show that observation of this athermal emission from multiple levels allows to obtain additional information about the relation 
between the levels, in particular whether or not they arise from the same defect. For this purpose, Cr implanted p-type Ge has been studied. Substitutional Cr can occur in 4 charge states in p-type Ge [8], corresponding to three defect levels: $\mathrm{Cr}^{+/ 0}\left(E_{A}=15\right.$ $\left.\mathrm{meV}, K_{T}=1.7 \times 10^{6} \mathrm{~s}^{-1} \mathrm{~K}^{-2}\right), \mathrm{Cr}^{0 /-}\left(E_{A}=46 \mathrm{meV}, K_{T}=3.9 \times 10^{7} \mathrm{~s}^{-1} \mathrm{~K}^{-2}\right)$ and $\mathrm{Cr}^{-/ 2-}$ $\left(E_{A}=92 \mathrm{meV}, K_{T}=1.6 \times 10^{8} \mathrm{~s}^{-1} \mathrm{~K}^{-2}\right)$. Right after the filling pulse $\mathrm{V}_{P}$, all $\mathrm{Cr}$ in the depletion region at $\mathrm{V}_{R}$ is in the $\mathrm{Cr}^{+}$-state. By gradual emission of 3 holes it evolves to the $\mathrm{Cr}^{2-}$-state. At $15 \mathrm{~K}$, thermionic emission from the $\mathrm{Cr}^{+}$level is so fast that it may be considered as instantaneous $\left(\mathrm{e}_{t h}^{-1}<<t_{0}\right)$ and the starting state for analysis is $\mathrm{Cr}^{0}$. In principle at even lower temperature the $\mathrm{Cr}^{+/ 0}$ transition can also be investigated, but as it is close to the hole freeze-out this was not attempted here. The DLTS spectrum of Cr-implanted Ge in the 15-55 $\mathrm{K}$ range is presented in figure 2(a). Even for a short $t_{W}(5 \mathrm{~ms})$ an extra emission component precedes the thermal peak of the $\mathrm{Cr}^{\mathrm{O} /-}$ level. At larger window times $(512 \mathrm{~ms})$ also below the thermal peak of the $\mathrm{Cr}^{-/ 2-}$ level an additional component is detected.

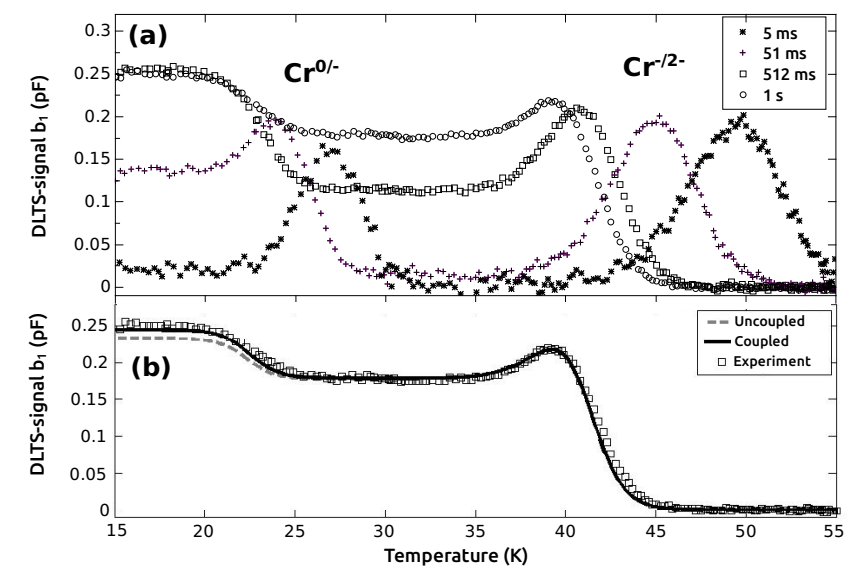

Figure 2. (a) DLTS spectrum of Cr in p-type Ge at different $t_{W}$. (b) DLTS spectrum for $t_{W}=1 \mathrm{~s}$ with spectrum simulations for the case of the coupled and uncoupled levels $\left(V_{R}=-1 \mathrm{~V}, V_{P}=-0.2 \mathrm{~V}\right)$.

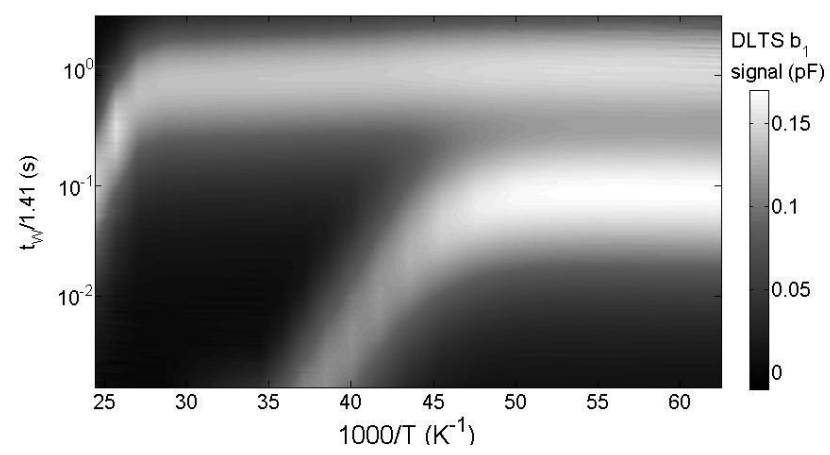

Figure 3. $t_{W}$ dependence of Ge:Cr. $V_{R}=-1 \mathrm{~V}, V_{P}=-0.2 \mathrm{~V}$. The ordinate represents $\mathrm{t}_{W} / 1.41$ in order to allow direct evaluation of $\tau_{\text {meas }}$. 
Like for Co, isothermal DLTS spectra have been recorded as a function of temperature and in figure 3 the $\mathrm{b}_{1}$-Fourier amplitude is plotted in grayscale as a function of window time and temperature. At $\mathrm{T}<20 \mathrm{~K}$, the DLTS spectrum is temperatureindependent. It is a superposition of two peaks with equal amplitude corresponding to non-thermal emission from the two considered $\mathrm{Cr}$ levels. The spectrum at these temperatures easily allows extracting the $\tau_{c}$ values, being $0.08 \pm 0.01 \mathrm{~s}\left(\mathrm{Cr}^{0 /-}\right)$ and $0.9 \pm 0.1 \mathrm{~s}\left(\mathrm{Cr}^{-/ 2-}\right)$. At higher temperatures $(20 \mathrm{~K}<\mathrm{T}<37 \mathrm{~K})$, the thermal emission rate of the first peak becomes important, thus shifting this peak towards lower $t_{W}$, while the amplitude does not change. The second peak, however, still stays at the same position up to $\mathrm{T}=37 \mathrm{~K}$. A further increase of the temperature causes also this second peak to shift towards lower window times, as the thermal emission rate becomes dominant also for that peak.

The analysis so far allows two interpretations of the observed spectra. The two DLTS peaks can arise from two different defects with the same (or very close) concentration(s) or from two levels of the same defect, e.g. 0/- and -/2- levels for Cr. Here we label these 2 possibilities as "uncoupled" and "coupled" respectively, referring to the rate equations describing them. When calculating the spectra of these two cases, a clear difference is obtained, which allows to distinguish between them, as discussed below. We first describe the situation of coupled levels as applies to the case of Cr.
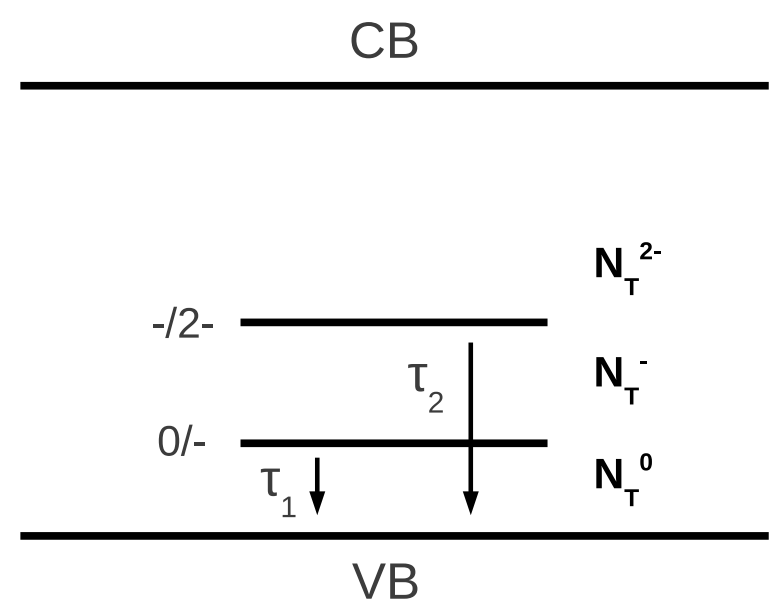

Figure 4. Model for hole emission from two acceptor levels related to the same defect.

As explained above, at the start of the capacitance transient measurements $\left(t_{0}\right.$ after the pulse) all Cr defects are assumed to be double occupied by holes $\left(\mathrm{Cr}^{0}\right)$. The subsequent hole emission processes are summarized in figure 4. The concentration of neutral centers $N_{T}^{0}$ decays by hole emission with emission time constant $\tau_{1}$

$$
\begin{aligned}
\frac{d}{d t} N_{T}^{0}(t) & =-\frac{1}{\tau_{1}} N_{T}^{0}(t) \\
\Rightarrow N_{T}^{0}(t) & =N_{T} e^{-t / \tau_{1}}
\end{aligned}
$$

As a consequence of the decay of $N_{T}^{0}$ by emission of holes, single negatively charged 
centers are formed, which at their turn disappear by emission of a second hole:

$$
\begin{aligned}
& \frac{d}{d t} N_{T}^{-}(t)=\frac{1}{\tau_{1}} N_{T}^{0}(t)-\frac{1}{\tau_{2}} N_{T}^{-}(t) \\
\Rightarrow & N_{T}^{-}(t)=N_{T} \frac{\tau_{2}}{\tau_{2}-\tau_{1}}\left(e^{-t / \tau_{2}}-e^{-t / \tau_{1}}\right)
\end{aligned}
$$

Finally, the concentration of defects in the double negative state is

$$
\begin{aligned}
& \frac{d}{d t} N_{T}^{2-}(t)=\frac{1}{\tau_{2}} N_{T}^{-}(t) \\
\Rightarrow & N_{T}^{2-}(t)=N_{T}\left[\frac{1}{\tau_{2}-\tau_{1}}\left(\tau_{1} e^{-t / \tau_{2}}-\tau_{2} e^{-t / \tau_{1}}\right)+1\right]
\end{aligned}
$$

The change in the charge density on the acceptors responsible for the measured capacitance transient (change of depletion width) in the observed window time is

$$
N_{T}^{-}(t)+2 N_{T}^{2-}(t)=2 N_{T}-\frac{N_{T}}{\tau_{2}-\tau_{1}}\left[\left(\tau_{2}-2 \tau_{1}\right) e^{-t / \tau_{1}}+\tau_{2} e^{-t / \tau_{2}}\right]
$$

For two uncoupled defect levels, on the other hand, the change in charge concentration on the acceptors is the sum of the two independent emission components:

$$
2 N_{T}-N_{T}\left[e^{-t / \tau_{1}}+e^{-t / \tau_{2}}\right]
$$

which have here been given the same $N_{T}$ trap concentration, as can be established from the ratio of their thermal peak amplitudes at small window times. The Fourier component DLTS signal $\mathrm{P}$ for the uncoupled system $\left(\mathrm{P}_{U}\right)$ is simply the sum of the two single DLTS signals:

$$
P_{U}=P_{1}+P_{2}
$$

For the coupled system, however, this is:

$$
P_{C}=(2-X) P_{1}+X P_{2}
$$

with

$$
X=\frac{\tau_{2}}{\tau_{2}-\tau_{1}}
$$

The computed relative difference between the system with coupled and uncoupled rate equations for different window times is shown in figure 5. Based on this figure, we can analyse the situation for Ge:Cr. It is reasonable to assume that $\tau_{1}$, the emission time constant for the shallower of the two defect levels, is at any temperature smaller than $\tau_{2}$, therefore only the coloured region of figure 5 is taken into account. A significant difference between the coupled and uncoupled model is observable above the white dashed line. At temperatures below $23 \mathrm{~K}$, both $\tau_{1}$ and $\tau_{2}$ are approximately equal to the (constant) emission times $\tau_{c, 1}(=0.08 \mathrm{~s})$ and $\tau_{c, 2}(=0.9 \mathrm{~s})$. The full white line in figure 5 corresponds to this situation for variable $t_{W}$.

For Cr, no significant difference between the coupled and uncoupled situation is predicted for $t_{W}=512 \mathrm{~ms}$. A marked difference between the coupled and uncoupled situation is expected for $t_{W}=5 \mathrm{~ms}, 51 \mathrm{~ms}$ and $1 \mathrm{~s}$. At the smaller window times, 


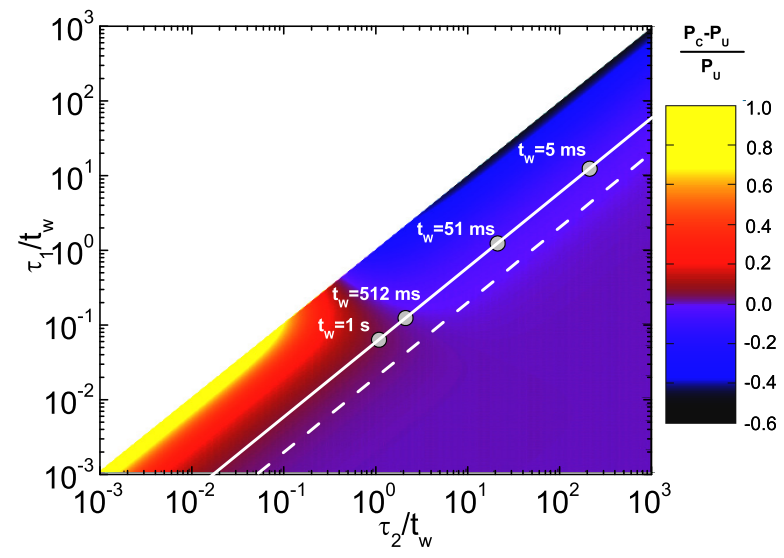

Figure 5. Relative intensity difference between the coupled and uncoupled system $\left(\mathrm{P}_{C}-\mathrm{P}_{U}\right) / \mathrm{P}_{U}$ (see text).

the additional components become small and although the relative difference between coupled and uncoupled cases is large, the measured absolute difference lies within the noise level. The difference between both models for $t_{W}=1 \mathrm{~s}$ is seen in figure 2(b) at temperatures below $23 \mathrm{~K}$. It is clear that the simulation for the coupled levels case fits the data much better: the two DLTS-peaks measured in the specimen with $\mathrm{Cr}$ arise from the same defect. One should remark that although the presence of non-thermal emission is not strictly required for making this distinction, it is necessary to observe emission from the two levels at the same temperature, which is greatly facilitated when it does occur.

\subsection{ORIGIN OF THE NON-THERMAL EMISSION}

The observed non-thermal emission can in principle originate from three sources: direct tunneling, recombination and photo-excitation. Each of these possibilities is discussed below.
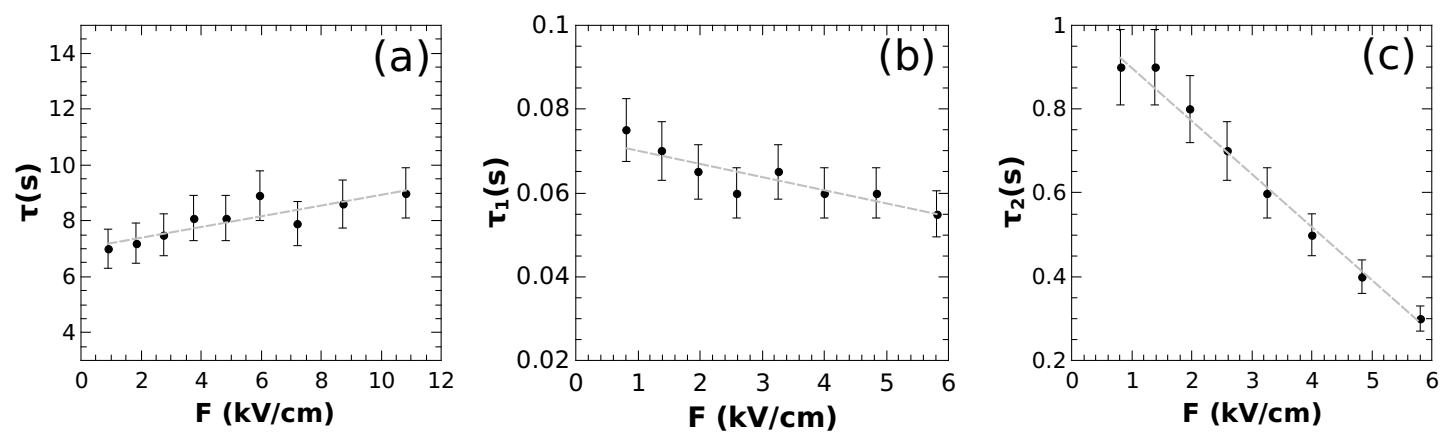

Figure 6. Temperature independent time constants of (a) $\mathrm{Co}^{+/ 0}$, (b) $\mathrm{Cr}^{0 /-}$ and (c) $\mathrm{Cr}^{-/ 2-}$ as a function of the electric field. 
In the DLTS spectra of certain quantum wells and dots a similar low-temperature non-thermal emission component has been observed and explained as the result of direct tunneling of a carrier out of the quantum well. [12 19] Other tunneling mechanisms that have been proposed for carriers out of defects or quantum wells, in particular phonon- and thermally assisted tunneling, are essentially thermally activated processes and cannot explain the current observations. In the case of direct tunneling, a strong dependence of $\tau_{c}$ on electric field is expected, as the field determines the width of the tunnel barrier and it may in addition lower the barrier height for attractive centers (Poole-Frenkel effect). For a square well potential one calculates [16]

$$
\tau^{-1}=\frac{e F}{4 \sqrt{2 m^{*} E_{i}}} \exp \left(-\frac{4}{3} \frac{\sqrt{2 m^{*}} E_{i}^{3 / 2}}{e \hbar F}\right)
$$

with $F$ the electric field, $E_{i}$ the binding energy, $m^{*}$ the effective mass and $e$ the elementary electric charge.

The electric field dependence for the three levels described in the previous subsections is shown in figure 6. It is obvious that none of these follow the expected strong field-dependence for direct tunneling. For $\mathrm{Co}^{+/ 0} \tau_{c}$ is practically independent of electric field, for $\mathrm{Cr}^{0 /-}$ it shows a weak dependence and for $\mathrm{Cr}^{-/ 2-}$ it is somewhat stronger. These dependences rather appear to follow the trend in Poole-Frenkel shift that these levels exhibit. [8, 9] Moreover the non-thermal emission is already observable at very low fields: in the case of a simple square well potential, the barrier width for hole tunneling from $\mathrm{Co}^{+}$at $1 \mathrm{kV} / \mathrm{cm}$ is estimated at $830 \mathrm{~nm}$, which seems unrealistic for allowing tunneling. Hence we may exclude direct tunneling from the defect level to the valence band as a mechanism.

Recombination with conduction band electrons also seems very unlikely, as it occurs in a layer depleted of free carriers. One might still consider recombination with electrons tunneling from interface states at the metal-semiconductor junction as a mechanism. [22] However, in all our experiments the minimal distance between the junction and the probed semiconductor region is at least $4 \mu \mathrm{m}$, rendering such a tunnel mechanism even less plausible than direct hole emission tunneling to the valence band.

For photo-excitation or -ionization to be observed, a sufficient flux of photons $\Phi$ of the right energy should be present. In that case, the release of holes from a defect with charge $\mathrm{q}$ at a temperature where thermal emission is negligible may be modeled as

$$
\frac{d N^{q}}{d t}=-\sigma_{P I} \Phi N^{q} \Rightarrow \tau_{c}=\left(\sigma_{P I} \Phi\right)^{-1}
$$

with $\sigma_{P I}$ the cross-section for photoionization. As origin of the photon flux one can consider black-body radiation from hotter parts of the cryostat, that is not completely blocked by the heat shields. For Ge:Co, an estimation of the size of the photo-excitation effect can been made. Barnik et al. have shown that photons with an energy between $100 \mathrm{meV}$ and $230 \mathrm{meV}$ have the largest contribution to the photoionization of Ge:Co (which is much larger than the activation energy of $83 \mathrm{meV}$ due to the donor nature of 
the $\mathrm{Co}^{+/ 0}$-level). 23] For a black body at $300 \mathrm{~K}$, a photon flux of $3.5 \times 10^{18} \mathrm{~cm}^{-2} \mathrm{~s}^{-1}$ is expected in this energy range. Considering the peak cross section for photoionization of $4.6 \times 10^{-16} \mathrm{~cm}^{2}[23]$, in the range of photoionization cross sections for other deep and shallow levels in Si and Ge $\left(10^{-17}-10^{-14} \mathrm{~cm}^{2}\right)$ [24 26], such fluxes may be sufficient to yield emission time constants in the order of $1 \mathrm{~s}$, as observed for the Co donor level, even if a partial blocking by the heat shields is taken into account. A black body at a considerably lower temperature of $200 \mathrm{~K}$ produces a photon flux of $2.8 \times 10^{17} \mathrm{~cm}^{-2} \mathrm{~s}^{-1}$ ), which may still be sufficient to yield temperature independent time constants in the right order of magnitude. In order to verify this possibility an experiment was carried out in which the sample space was covered by aluminium foil, as an extra heat shield.

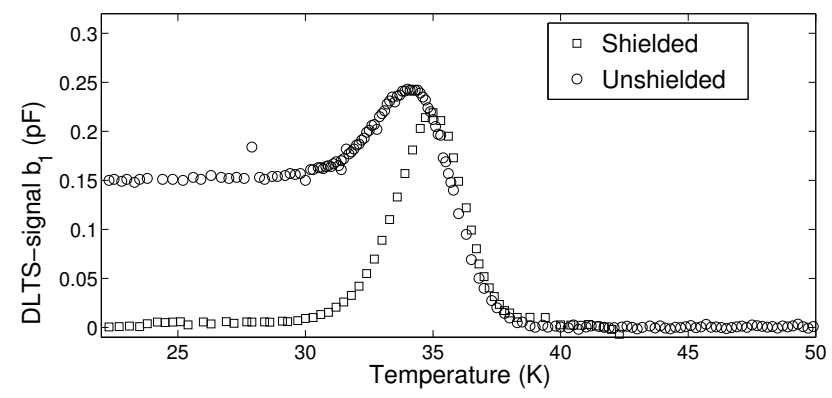

Figure 7. DLTS spectra of Ge:Co with (Shielded) and without (Unshielded) aluminium foil cover

The results of a measurement with and without this additional heat shield are shown in figure 7, clearly demonstrating that the non-thermal emission component can indeed be assigned to photoionization by black-body radiation in the cryostat.

The fact that the time constant for hole emission at low temperature is independent of temperature, demonstrates that this effect is dominated by photoionization and that photothermal ionization plays an insignificant role. Related to this, it has been shown that the oscillator strength for internal transitions in shallow donors and acceptors in Ge decreases with increasing binding energy. 27] If the latter also applies to the photoionisation transitions, it may explain the absence of non-thermal emission from the deeper $\mathrm{Co}^{0 /-}$ level, together with a lower photon flux above $254 \mathrm{meV}$. It is reasonable to assume that the same mechanism applies to Ge:Cr. For these acceptor levels the peak in $\sigma_{P I}$ is expected to lie much closer to the activation energy for thermal emission, which explains why observed $\tau_{c}$ values are considerably smaller. In spite of considerable efforts we were unable to identify the source of the black-body radiation in the cryostat and hence its spectrum (effective black body temperature) remains unknown. As a result, we were unable to extract $\sigma_{P I}$ values for the $\mathrm{Cr}$ acceptor levels via comparison of their $\tau_{c}$ values with that of the Co donor level.

Finally, the determination of the carrier cross-section in section 3.1- in the presence of a source of radiation, not taken into account in previous analyses - deserves some further attention. The method used to analyze the increase of the DLTS signal as 
a function of filling pulse not only takes into account the slow capturing regime but also the effect of emission. This correction may thus also include an emission rate due to photoionization, as long as it remains much slower than the observed capture rate $\left(c_{p} p\right)$. The requirement in the model that the capture rate is faster than the emission rate, is automatically fulfilled (by thermodynamics) in the absence of photoionization, and remains valid with photoionization at this low light intensity. Since we have experimentally observed that $c_{p} p \gg 1 / \tau_{c}\left(c_{p} p \sim 106 \mathrm{~s}^{-1}, e_{c} \sim 0.14 \mathrm{~s}^{-1}\right)$, no effect on the determination of the capture cross-section is expected.

\section{CONCLUSIONS}

In this paper, we showed via DLTS experiments on transition-metal doped Ge that in addition to thermal emission, a second, non-thermally activated path for carrier emission from deep-level defects may exist. Photo-ionization by far-infrared photons due to black-body radiation in the cryostat lies at the origin of this extra emission pathway. We observed this non-thermal emission as a low-temperature tail in the DLTS spectra for the $\mathrm{Co}^{+/ 0}, \mathrm{Cr}^{0 /-}$ and $\mathrm{Cr}^{-/ 2-}$ levels in Ge. As the spectrum of the radiation is not known, no direct information on the photo-ionization of the Cr acceptor levels can be obtained from comparison with the known values for the Co donor level. The nonthermal emission does allow to determine carrier capture cross-sections for the defect states at low temperature and to establish unambiguously whether or not defect levels, for which it is observed, belong to the same defect.

\section{Acknowledgments}

The authors like to thank Umicore for providing the Ge samples investigated in this work and the FWO for financial support through grant $\mathrm{n}^{\circ}$ G.0207.10N. We thank the reviewers for their contributions to elucidating the non-thermal emission mechanism.

\section{References}

[1] Lang D V 1974 J. Appl. Phys. 453023

[2] Simoen E, Clauws P, Lamon M and Vennik J 1986 Semicond. Sci. Technol. 1 53-57

[3] Simoen E, Clauws P, Huylebroeck G and Vennik J 1987 Semicond. Sci. Technol. 2 507-512

[4] Kamiura Y and Hashimoto F 1989 Jap. J. Appl. Phys. 28 763-769

[5] Clauws P and Simoen E 2006 Mater. Sci. Semicond. Process. 9 546-553

[6] Forment S, Vanhellemont J, Clauws P, Van Steenbergen J, Sioncke S, Meuris M, Simoen E and Theuwis A 2006 Mater. Sci. Semicond. Process. 9 559-563

[7] Clauws P, Van Gheluwe J, Lauwaert J, Simoen E, Vanhellemont J, Meuris M and Theuwis A 2007 Physica B 401-402 188-191

[8] Lauwaert J, Van Gheluwe J, Vanhellemont J, Simoen E and Clauws P 2009 J. Appl. Phys. 105 073707

[9] Lauwaert J, Vanhellemont J, Simoen E, Vrielinck H and Clauws P 2012 J. Appl. Phys. 111113713

[10] Gurimskaya Y, Mathiot D, Sellai A, Kruszewski P and Dobaczewski L $2011 \mathrm{~J}$. Appl. Phys 110 113707 
[11] Claeys C and Simoen E (eds) 2007 Germanium-Based Technologies (Elsevier)

[12] Vincent G, Chantre A and Bois D 1979 J. Appl. Phys. 50 5484-5487

[13] Letartre X, Stievenard D, Lannoo M and Barbier E 1991 J. Appl. Phys 69 7336-7338

[14] Kapteyn C M A, Heinrichsdorff F, Stier O, Heitz R, Grundmann M, Zakharov N D, Bimberg D and Werner P 1999 Phys. Rev. B 60 14265-14268

[15] Geller M, Stock E, Kapteyn C, Sellin R L and Bimberg D 2006 Phys. Rev. B 73205331

[16] Schulz S, Schramm A, Heyn C and Hansen W 2006 Phys. Rev. B 74033311

[17] Engstrom O, Kaniewska M, Kaczmarczyk M and Jung W 2007 Appl. Phys. Lett. 91133117

[18] Schramm A, Schulz S, Heyn C and Hansen W 2008 Phys. Rev. B 77153308

[19] Schramm A, Schulz S, Zander T, Heyn C and Hansen W 2009 Phys. Rev. B 80155316

[20] Blood P and Orton J W 1992 The electrical characterization of semiconductors: majority carriers and electron states (Academic press)

[21] Lauwaert J, Van Gheluwe J and Clauws P 2008 Rev. Sci. Instr. 79093902

[22] Schmalz K, Yassievich I N, Rucker H, Grimmeiss H, Frankenfeld H, Mehr W, Osten H J, Schley P and Zeindl H P 1994 Phys. Rev. B 19 14287-14301

[23] Barnik M I, Beglov B I, Romanychev D A and Kharionovskii Y S 1971 Sov. Phys. -Semicond. 5 $87-91$

[24] Braun S, Grimmeiss H G and Spann K 1977 J. Appl. Phys. 483883

[25] Jungwirt G and Prettl W 1991 Infrared Phys. 32 191-194

[26] Pässler R, Pettersson H, Grimmeiss H G and Schmalz K 1997 Phys. Rev. B 55 4312-4322

[27] Rotsaert E, Clauws P, Vennik J and Van Goethem L 1989 J. Appl. Phys. 65730 